\title{
Leben mit Myasthenie
}

Dieses Heft von psychoneuro informiert als Themenschwerpunkt über die Myasthenie als seltene neuromuskuläre Erkrankung mit maximal 12000 Erkrankten in Deutschland. Immer mehr Myastheniepatienten kann heute eine Therapie angeboten werden, die ein nahezu unbeeinträchtigtes Leben ermöglicht. In den Schwerpunktbeiträgen finden sich jedoch auch sehr bemerkenswerte Hinweise, dass Myastheniepatienten im sich rasch wandelnden Gesundheitssystem zunehmend schwieriger eine ausreichende Hilfe finden. Dies gilt sicher auch für andere Patientengruppen mit seltenen oder sehr seltenen Erkrankungen.

Zunehmend werden neue Immunsuppressiva bei der Myasthenietherapie eingesetzt, worüber S. Jander berichtet. Sie sind eine wichtige Option für die Untergruppe von Myastheniekranken, die nicht ausreichend auf die hergebrachten Therapien reagieren. Kontrollierte Studien sind schon wegen der Seltenheit und dem sehr unterschiedlichen Verlauf der Myasthenie kaum durchführbar. Trotz der Schwere der häufig lebensbedrohlichen Erkrankung verweigern gesetzliche Krankenkassen jedoch immer wieder eine Kostenübernahme mit Hinweis auf eine vermeintlich nicht ausreichende Studienlage. Im Beitrag zum Pyridostigmin, das seit mehr als 50 Jahren die Basis der Myastheniebehandlung ist, wird beschrieben, wie die Acetylcholinesteraseinhibitoren in die Therapie eingeführt wurden. Die englische Neurologin Mary Walker hatte 1934 intuitiv Ähnlichkeiten zwischen der Myasthenie und der Wirkung von Curare erkannt. Der verblüf- fende Effekt des darauf von ihr bei einer einzelnen Patientin eingesetzten Physostigmin wurde direkt in „Lancet“ publiziert. Studien wurden selbstredend damals nicht unternommen. Ein Wirkungsnachweis nach den Prinzipien der Evidenz-basierten Medizin fehlt weiterhin, obwohl niemand ernsthaft behaupten wird, dass Acetylcholinesteraseinhibitoren nicht hochwirksam seien.

Die Myasthenie ist eine Erkrankung, deren Therapie individuell ausgerichtet werden muss. Leitlinien können nur Orientierungspunkte liefern, jedoch nie die Einschätzung des in der Myasthenietherapie erfahrenen Neurologen ersetzen. Viele Myastheniepatienten erhalten jedoch keine angemessene Therapie. Weiterhin kommt es zu myasthenen Krisen durch Therapiefehler oder es wird in besonderen Situationen, wie bei Kinderwunsch und Schwangerschaft, nicht adäquat reagiert. Immer noch gibt es in Deutschland keine zertifizierten Myastheniezentren, deren Etablierung dringend erforderlich ist.

Hilfreich für alle Myastheniepatienten ist eine Mitgliedschaft in der Deutschen MyasthenieGesellschaft in Bremen (www.dmg-online.de). Diese sollten Sie all Ihren Myastheniepatienten unbedingt nahe legen.

Ganz herzlich lade ich Sie zu unserem 3. Stralsunder Neurophysiologie-Wochenende vom 3.-5.10.2007 an die Ostsee ein. Ein Thema dieses EMG-Kurses wird die elektrophysiologische Myastheniediagnostik sein. Das Programm schicken wir Ihnen gerne zu (j.sieb@klinikum-hst.de).

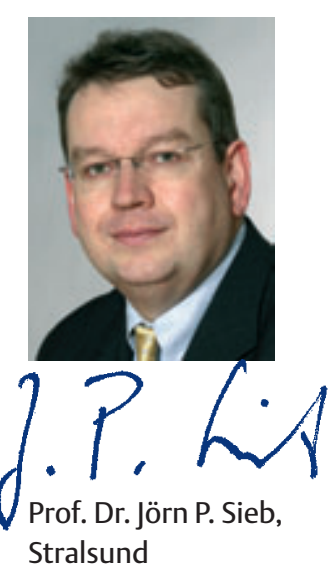

Stralsund 